bacterial meningitis. The editors state that their intention was to produce a state-of-the-art monograph dealing with various aspects of meningitis, and several of the papers provide very detailed and useful reviews of current aspects of epidemiology, pathogenesis, diagnosis and therapy of bacterial meningitis, with a leaning towards North American experience. The papers dealing with cerebrospinal fluid penetration of antimicrobials and the pathogenesis of central nervous system injury in bacterial meningitis are particularly interesting and provide much information. Most papers are extensively referenced and an index is included. Inevitably, with works from several authors, there is some duplication of information, particularly relating to epidemiology, but generally this is not excessive.

The therapy of meningitis is dealt with in several papers with discussions on choice of antibiotic, dosage, duration of treatment and use of adjunctive agents including steroids, non-steroidal anti-inflammatory drugs and immunological agents such as antibacterial antibodies, anti-cytokine antibodies and the leucocyte-directed-agent, pentoxifylline. It is clear from these reviews that there is no consensus of opinion on optimum treatment, and that much more work is needed to define the precise role of the newer agents in the clinical setting.

The English in one or two of the papers is a little unconventional (no doubt as a result of translation), and this can make interpretation of the text difficult.

Generally this book provides an interesting and stimulating volume which fulfils the editorial intention. There is something here for all who come across this challenging disease, although perhaps those without a particular interest may find a general text more appropriate, especially given the price of the volume.

C. Catchpole

\section{Control of Hospital Infection-a Practical Handbook}

Edited by G. A. J. AylifFe, E. J. L. Lowbury, A. M. GEDDES, J. D. WILLIAMS. 1992. ISBN 0-412-28440-5. Chapman and Hall Medical, London. Pp. 381. £45.00.

The publication of the third edition of Control of hospital infection-a practical handbook will be welcomed by all who work in the field of infection control.

The presentation in this edition is much improved by the change in type face and use of a better quality paper. The content and presentation will be immediately recognisable to those who have read and used the previous two editions of this "bible" of infection control. Although much of the content and advice remains the same, this edition incorporates changes that have occurred in the 10 years since the last edition. The emergence of HIV and AIDS resulted in their inclusion into a chapter devoted solely to "The control of viral hepatitis and human immunodeficiency (HIV) infection". Other diseases and organisms which appear for the first time or receive increased coverage include: EMRSA, pseudomembranous colitis, legionella, the viral haemorrhagic fevers and cryptosporidiosis.

A worthwhile addition to the section on surveillance and reporting of infection is a list of definitions of hospitalacquired infection applicable to different body systems. The adoption of these definitions could lead to easier comparison of infection rates between reporting centres. Unfortunately a definition for wound infection is missing.

The Control of Substances Hazardous to Health (COSHH) Regulations are briefly covered, especially with respect to the chemical hazards associated with glutaraldehyde and ethylene oxide. Advice on the assessment of the hazards caused by microbes in the clinical setting is lacking, possibly due to the editors' uncertainty concerning the Health and Safety Executive application of $\mathrm{COSHH}$ Regulations to microbial infection at ward level. The possession of control of infection policies is not sufficienthazard assessments may also be required.

Compliance with regulations for the disposal of clinical waste, including its incineration, is currently presenting problems for hospitals. The section covering this subject would have been better as a separate chapter, or as part of the chapter on responsibilities, rather than sandwiched within the section on kitchen hygiene. Kitchen hygiene and inspection is comprehensively covered but advice on ward kitchen hygiene or the microbiologically safe use of microwaves is lacking.

This handbook is one of the few which deal comprehensively with all aspects of infection control. It succeeds admirably in its aim of being a practical manual and its users will find answers to most of the questions posed to Infection Control Team members. Indeed, the advice in this manual probably forms the core of many of the control of infection policies currently employed in hospitals in the UK and even further afield. It is essential reading for trainees in medical microbiology, public health medicine and for Infection Control Nurses, as well as being a reference work for Control of Infection Doctors and other health care managers.

M. F. NoY

Viral Warts. Biology and Treatment, 2nd edition.

Mary H. Bunney, Clatre Benton, Heather A. Cubie. 1992. ISBN 0-19-262062-2. Oxford University Press. Pp. 180. $£ 45.00$.

This is the second edition of a most useful text on viral warts. The first edition was written by Dr Bunney in 1982 . Since then there has been an enormous increase in interest in the biology, molecular biology and pathogenicity of papilomaviruses. The first edition has been extensively revised to take account of this and two new authors have been coopted.

The book is subdivided into three sections. The first section deals with the virology, molecular biology, immunology and pathogenicity of papillomaviruses. It provides an excellent introduction to the topic, covering (with illustrations) new techniques, such as Southern blotting, dotblotting, in-situ hybridisation and DNA amplification by PCR, for detecting and typing papillomaviruses. Some guidance on the relative value of these techniques is also included. Other chapters cover the immunology and immunity to wart viruses, and pathogenesis. The other two sections cover the diagnosis, clinical features, management and presentation of cutaneous and mucocutaneous warts and include useful clinical information.

The book is hard-backed and cloth-bound on good paper and contains many well-reproduced illustrations in both colour and black and white. It is written in a way that is easily accessible for readers of differing technical and clinical proficiency. I am sure it will be a useful addition to libraries in dermatology, genitourinary medicine and virology departments.

C. A. HART 